\title{
Theoretische Behandlung des Einflusses sterischer Effekte auf die Reaktivität aliphatischer Verbindungen
}

\author{
II. Zum Orientierungsproblem bei bimolekularen Abspaltungsreaktionen \\ Von FrIEDRICH BeCKer \\ Aus dem Institut für Physikalische Chemie der Universität des Saarlandes, Saarbrücken \\ (Z. Naturforschg. 15 b, 251-260 [1960]; eingegangen am 31. Oktober 1959)
}

\begin{abstract}
Die in der 1. Mitteilung beschriebene Methode wird auf das Problem der Orientierung bei bimolekularen Abspaltungsreaktionen angewandt. Eine Untersuchung der sterischen Wirksamkeit von Vergrößerungen des Raumbedarfs der Alkylreste $R$ an der entstehenden Doppelbindung, der als Reaktionspartner im Übergangszustand angreifenden Base und der abzuspaltenden elektrophilen Gruppe X auf das Verhältnis von 1- und 2-Olefin im Reaktionsprodukt führt zu folgenden Ergebnissen :

1. Maßgeblich für die sterischen Einflüsse auf die Orientierung sind Abstoßungen zwischen den Alkylresten $R$ und der Base, die im Übergangszustand für das 2-Olefin überwiegen.

2. Eine Zunahme des Raumbedarfs der Base verursacht eine systematische Verschiebung des Isomerenverhältnisses zugunsten des 1-Olefins.

3. Kettenverlängerungen und -verzweigungen des Alkylrestes $R$ sind nur dann sterisch wirksam, wenn sie von dem zur elektrophilen Gruppe $\mathrm{X} \gamma$-ständigen C-Atom ausgehen.

4. Der Raumbedarf der elektrophilen Gruppe $X$ übt keinen sterischen Einfluß auf das Isomerenverhältnis aus.
\end{abstract}

Wie in der 1 . Mitteilung ${ }^{1}$ gezeigt wurde, kann das Problem des Einflusses sterischer Effekte auf die Reaktivität aliphatischer Verbindungen auf eine Bestimmung der sterischen Verteilungsfunktionen von Ausgangs- und Übergangszuständen der zu vergleichenden Reaktionen zurückgeführt werden. Die hierzu erforderlichen sterischen Verteilungsfunktionen lassen sich mit Hilfe des beschriebenen Abzählverfahrens für die Energien der Konformationen ermitteln.

\section{Allgemeine Gesichtspunkte bei der Anwen- dung der sterischen Verteilungsfunktion auf Reaktivitätsprobleme}

Bei den Ausgangszuständen der zu betrachtenden Reaktionen handelt es sich um stabile Moleküle von bekannter Struktur, die sich von den in der 1. Mitteilung ${ }^{1}$ untersuchten Paraffinen durch die Anwesenheit funktioneller Gruppen unterscheiden. Der für sterische Effekte maßgebende Raumbedarf dieser Gruppen kann an Hand ihrer Wirkungsradien abgeschätzt werden. Der einfachste Weg für die Aufstellung der sterischen Verteilungsfunktion besteht darin, daß man rein formal die funktionelle Gruppe durch eine Alkylgruppe von möglichst gleichem Raumbedarf ersetzt und die sterische Verteilungsfunktion des erhaltenen Kohlenwasserstoffs verwendet. Ob ein solches Vorgehen eine brauchbare Näherung darstellt, muß von Fall zu Fall geprüft werden; Abweichungen im Raumbedarf lassen sich gegebe- nenfalls durch besondere Abstoßungsparameter zwischen den funktionellen Gruppen und den übrigen Substituenten des Moleküls berücksichtigen. Es kommt vor allem darauf an, daß der gesuchte sterisch äquivalente Kohlenwasserstoff hinsichtlich der Valenzwinkel und der Zahl der Bindungen mit gehemmter innerer Rotation mit dem gegebenen Molekül übereinstimmt.

Für den instabilen Übergangszustand der Reaktionen muß ein Modell verwendet werden, das mit den Ergebnissen reaktionskinetischer Untersuchungen im Einklang steht; der Anwendungsbereich der Methode ist somit auf Reaktionen beschränkt, für die sich ein genügend gesichertes geometrisches Modell des Übergangszustandes aufstellen läßt. Grundsätzlich enthält der Übergangszustand bimolekularer Reaktionen den Reaktionspartner in einem Abstand vom Reaktionszentrum, der von der GröBenordnung normaler Atomabstände ist, so daß dieser gewissermaßen die Rolle eines neuen Substituenten übernimmt. Da es für die sterischen Effekte nur auf den Raumbedarf ankommt, kann man auch hier versuchen, den Reaktionspartner formal durch einen sterisch äquivalenten Alkylsubstituenten zu ersetzen. Falls erforderlich, läßt sich das Modell durch die Einführung besonderer Abstoßungsparameter zwischen dem Reaktionspartner und den Substituenten des Ausgangsmoleküls verfeinern. Die Tatsache, daß man sich hierbei mangels genauer empirischer

1 F. BeCKer, Z. Naturforschg. 14 a, 547 [1959]. 
Unterlagen meistens mit ziemlich groben Abschätzungen begnügen muß, bedeutet keinen schwerwiegenden Nachteil, da die qualitativen Aussagen der Theorie über die grundsätzliche Wirksamkeit und die Herkunft der strukturellen sterischen Effekte von den verwendeten Zahlenwerten für die Abstoßungsparameter weitgehend unabhängig sind. Außerdem muß berücksichtigt werden, daß von den intramolekularen Abstoßungskräften nur diejenigen Unterschiede zwischen Ausgangs- und Übergangszustand einen Einfluß auf die relativen Geschwindigkeitskonstanten haben, die sich innerhalb einer Vergleichsserie von Reaktion zu Reaktion ändern.

Die zur Berechnung der sterischen Verteilungsfunktion notwendige Zurückführung von Ausgangsund Übergangszuständen auf verzweigte Paraffinmoleküle schränkt den Anwendungsbereich der Methode auf solche Fälle ein, in denen die Liganden am Reaktionszentrum im Übergangszustand tetraedrisch angeordnet sind.

\section{Die Orientierungsregeln von $H$ of $m$ a $n$ und $S$ a y $t z$ e f f für Abspaltungsreaktionen}

Zur Prüfung der Brauchbarkeit der entwickelten Theorie sind die olefinbildenden Abspaltungsreaktionen besonders geeignet, weil ihr Reaktionsmechanismus weitgehend aufgeklärt ist, und verhältnismäßig viele experimentelle Daten über den Einfluß struktureller Effekte auf die Orientierung zur Verfügung stehen.

Nach den Untersuchungen von Hughes, Ingold und Mitarbb. ${ }^{2}$ kommen für Abspaltungsreaktionen in Lösung zwei verschiedene Mechanismen in Betracht: Der bimolekulare Mechanismus (E 2), bei welchem der Prozeß in einem einzigen Schritt abläuft und der unimolekulare Mechanismus (E 1), bei dem die Reaktion in zwei Stufen erfolgt. Im ersteren Fall ist ein nukleophiles Reagenz B, gewöhnlich eine Base, erforderlich, die mit Hilfe ihres freien Elektronenpaars der Verbindung HX ein H-Atom als Proton zu entreißen vermag:

$$
\mathrm{B}:+\mathrm{H}-\mathrm{CR}_{2}-\mathrm{CR}_{2}-\mathrm{X} \rightarrow \mathrm{BH}+\mathrm{CR}_{2}=\mathrm{CR}_{2}+: \mathrm{X} \text {. }
$$

Dieser Vorgang wird durch die gleichzeitige Los-

2 M. L. Dhar, E. D. Hughes, C. K. Ingold, A. M. M. Mandour, G. A. Maw u. L. I. Woolf, J. chem. Soc. [London] 1948, 2093 ; C. K. IngoLd, „Structure and Mechanism in Organic Chemistry“, Cornell University Press, Ithaca 1953, S. 419 bis 472 .

3 A. W. Hofmann, Liebigs Ann. Chem. 78, 253 [1851]; 79, 11 [1851].

4 A. Saytzeff, Liebigs Ann. Chem. 179, 296 [1875]. lösung der elektrophilen Gruppe X unter Mitnahme des bindenden Elektronenpaars und durch die Schließung der Doppelbindung unterstützt. Hat man es mit unsymmetrischen sekundären oder tertiären Alkylderivaten $\mathrm{zu}$ tun, so können verschiedene isomere Olefine nebeneinander entstehen, wie z. B. aus 2-Brombutan:

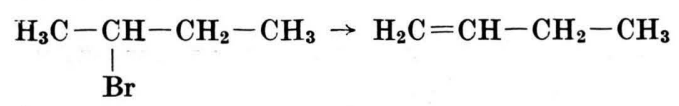

2-Brombutan<smiles>C/C=C\CCC</smiles>

Gewöhnlich ist in solchen Fällen eine der Abspaltungsrichtungen bevorzugt, wobei stukturelle Einflüsse eine maßgebende Rolle spielen. Nach einer von HoFmanN $^{3} 1851$ aufgestellten Orientierungsregel erhält man bei der Einwirkung von Basen auf quartäre Ammoniumsalze vorzugsweise das Olefin mit den wenigsten Alkylsubstituenten an der Doppelbindung. SAytZEFF ${ }^{4}$ hingegen fand 1875, daß die Halogenwasserstoff-Abspaltung aus sekundären und tertiären Alkylhalogeniden überwiegend das Olefin mit den meisten Alkylgruppen an der Doppelbindung liefert. Wie Hughes, Ingold und Mitarbb. ${ }^{2}$ später feststetlten, ist der Anwendungsbereich der $\mathrm{H}$ of m a n n schen Regel auf die bimolekulare Abspaltung aus Oniumverbindungen beschränkt, während die $\mathrm{S}$ a y $\mathrm{tz}$ e f $\mathrm{f}$ sche Orientierungsregel für alle übrigen bimolekularen Abspaltungsprozesse sowie generell für die unimolekulare Abspaltungsreaktion gilt.

Zur Deutung dieser beiden scheinbar einander widersprechenden Orientierungsregeln nahmen Hughes und INGold an, daß der beobachtete strukturelle Einfluß auf die Orientierung das Ergebnis einer Überlagerung von Hyperkonjugations- und Induktionseffekt darstellt. Nach der Auffassung dieser Autoren läßt die Saytzeff sche Regel das Überwiegen des Hyperkonjugations-Einflusses der Alkylgruppen an der entstehenden Doppelbindung erkennen, während das Vorherrschen des Induktionseinflusses der elektrophilen Gruppe X im Übergangszustand der bimolekularen Abspaltungsreaktion zu einer Orientierung nach der $\mathrm{H}$ o f $m$ a $n$ n schen Regel führt. Für die Wirksamkeit sterischer Effekte ergab sich aus den Untersuchungen von Hughes und INGOLD kein besonderer Anhaltspunkt. 
In den letzten Jahren konnten jedoch Brown und Mitarbb. ${ }^{5}$ zeigen, daß unter bestimmten strukturellen Voraussetzungen auch sterische Einflüsse auf die Orientierung der Abspaltung Bedeutung erlangen. Diese sterischen Effekte bewirken eine Verschiebung des Isomerenverhältnisses im Sinne der $\mathrm{H}$ o f m a n n schen Regel und haben ihre Ursache nach der Ansicht von BRown

1. im Raumbedarf der Alkylreste $\mathrm{R}$ an der entstehenden Doppelbindung,

2. im Raumbedarf der als Reaktionspartner an den $\mathrm{H}$-Atomen angreifenden Base B,

3. im Raumbedarf der abzuspaltenden elektrophilen Gruppe X.

Brown folgerte aus den Ergebnissen seiner Arbeiten, daß für eine Orientierung der Abspaltung nach der $\mathrm{H}$ of $\mathrm{m}$ a $\mathrm{n}$ schen Regel in erster Linie sterische Effekte maßgebend seien, während er dem Induktionseffekt nur eine untergeordnete Rolle beimißt. Tatsächlich zeichnen sich auch die nach $\mathrm{Hu}$ ghe s und Ing old besonders stark induktiv wirksamen Oniumverbindungen gleichzeitig durch einen erhöhten Raumbedarf in unmittelbarer Nähe der entstehenden Doppelbindung aus.

Alle bisher gegebenen Deutungen der Orientierungsregeln bei Abspaltungsreaktionen haben rein qualitativen Charakter und erlauben keine quantitativen Aussagen über den Anteil hyperkonjugativer, induktiver oder sterischer Einflüsse am beobachteten Gesamteffekt. Es war daher von Interesse, eine Klärung der noch offenstehenden Probleme mit Hilfe der Methode der sterischen Verteilungsfunktion zu versuchen.

\section{Das Modell für den Übergangszustand der bimolekularen Abspaltungsreaktion}

Nach den Untersuchungen von HüCKEL ${ }^{6}$, BARTON $^{7}$ und CRistor ${ }^{8}$ besitzt die bimolekulare Abspaltungsreaktion eine Stereospezifität, und zwar hat die Aktivierungsenergie für die heterolytische Reaktion in Lösung ihren niedrigsten Wert, wenn die angreifende Base B, das abzuspaltende Proton, die beiden C-Atome der entstehenden Doppelbindung und die abzuspaltende elektrophile Gruppe $\mathrm{X}$ eine ebene trans-Konfiguration einnehmen:

5 H. C. Brown u. Mitarbb., J. Amer. chem. Soc. 78, 2190 [1956] u. ff.

6 W. Hückel, W. Tappe u. G. Legutke, Liebigs Ann. Chem. 543, 191 [1940].

7 D. H. R. Barton u. E. Miller, J. Amer. chem. Soc. 72, 1066 [1950].

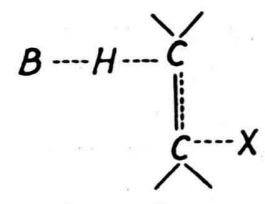

Abweichungen von dieser bevorzugten trans-Elimination werden nur beobachtet, wenn die Einstellung der hierfür günstigsten Konformation an der entstehenden Doppelbindung durch Einbau der betreffenden Gruppierung in ein enggliedriges Ringsystem behindert ist ${ }^{9}$. Bei den hier zur Diskussion stehenden offenkettigen Verbindungen ist aber mit einer solchen Behinderung nicht zu rechnen, so daß für den Übergangszustand nur diejenigen Konformationen in Betracht kommen, welche die Voraussetzungen für eine ebene trans-Elimination erfüllen.

Als Beispiel sei die auf S. 248 formulierte bimolekulare $\mathrm{HBr}$-Abspaltung aus 2-Brombutan betrachtet. Die Konformationen bezüglich der Bindung $\mathrm{C}_{2}-\mathrm{C}_{3}$ geben die Verhältnisse für die Bildung des transund cis-2-Butens wieder:

Konformationen des Ausgangszustandes:
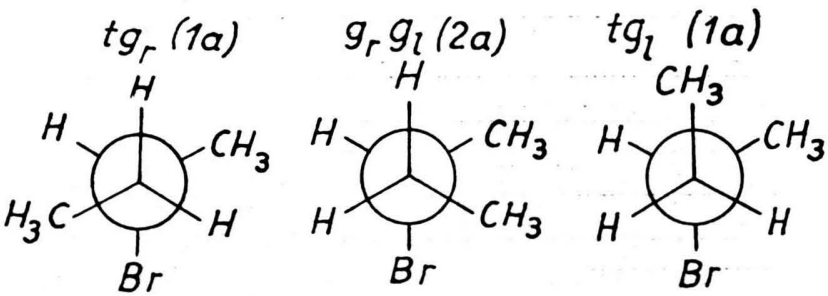

Konformationen der Übergangszustände:

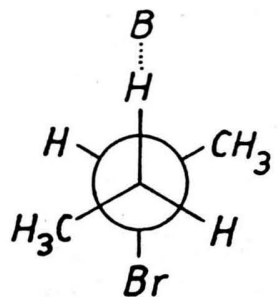

trans2-Buten

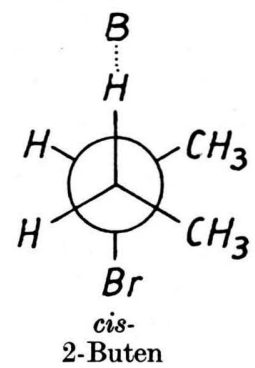

Von den 3 Konformationen des Ausgangszustandes erfüllen nur $\operatorname{tg}_{\mathrm{r}}$ und $\mathrm{g}_{\mathrm{r}} \mathrm{g}_{\mathrm{e}}$ die Bedingung der transStändigkeit von $\mathrm{H}$ und $\mathrm{Br}$ ( $\mathrm{zu}$ den Bezeichnungen der Konformationen vgl. die 1 . Mitteilung ${ }^{1}$ ). Greift die Base B am trans-ständigen H-Atom der Konfor-

8 S. J. Cristor, J. Amer. chem. Soc. 69, 338 [1947] ; 71, 1894 [1949] ; S. J. Cristol, N. L. Hause u. J. S. Meek, J. Amer. chem. Soc. 73, 674 [1951].

9 S. J. Cristol u. N. L. Hause, J. Amer. chem. Soc. 74, 2193 [1952]. 
mation $\operatorname{tg}_{\mathrm{r}}$ an, so erhält man den Übergangszustand für das trans-2-Buten, während die Konformation $\mathrm{g}_{\mathrm{r}} \mathrm{g}_{\mathrm{e}}$ den Übergangszustand für das cis-2-Buten liefert. Jeder dieser Übergangszustände besitzt demnach nur 1/3 der Konformationen einer normalen Verbindung von gleicher Kettenlänge.

Für die Bildung des 1-Butens sind die 3 identischen Konformationen bezüglich der Bindung $\mathrm{C}_{1}-\mathrm{C}_{2}$ maßgebend:
(3 identische)

Konformationen des

Ausgangszustandes :

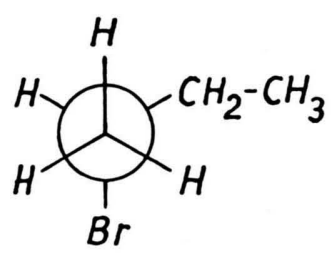

Konformation des Ubergangszustandes für das l-Buten:

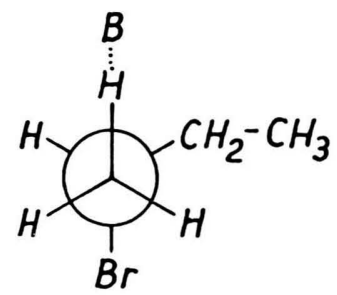

Da sie sämtlich die Bedingungen für eine transElimination erfüllen, erhält der betreffende Übergangszustand das statistische Gewicht 3.

Bei diesen Betrachtungen wurde angenommen, daß die tetraedrische Anordnung der Liganden an den beiden C-Atomen, zwischen denen die Doppelbindung entsteht, auch im Übergangszustand im wesentlichen erhalten bleibt. Gegen die Annahme einer nahezu koplanaren Anordnung dieser Liganden lassen sich mehrere Gründe anführen. In diesem Falle hätte man nämlich im Übergangszustand bereits ähnliche sterische Verhältnisse wie in den fertigen Olefinen vorliegen und daher entsprechende Energieunterschiede zwischen den trans- und cis-Isomeren zu erwarten. In einigen Fällen ist es möglich (z. B. bei den Isomerenpaaren $\mathrm{R}-\mathrm{CH}_{2}-\mathrm{C}\left(\mathrm{CH}_{3}\right)$ $=\mathrm{CH}_{2}$ und $\mathrm{R}-\mathrm{CH}=\mathrm{C}\left(\mathrm{CH}_{3}\right)_{2}$ mit $\mathrm{R}=$ Äthyl-, Isopropyl- und $t$-Butyl-), die Unterschiede der sterischen Energien aus den gemessenen Hydrierungswärmen zu errechnen ${ }^{10}$. Hierbei ergeben sich jedoch Energiebeträge, die viel zu groß sind, um das beobachtete Isomerenverhältnis bei den betreffenden Abspaltungsreaktionen (vgl. Tab. 2) richtig wiedergeben zu können, selbst wenn man den zusätzlichen, vom Raumbedarf der Base im Übergangszustand herrührenden sterischen Effekt unberücksichtigt läßt. Auch spricht die Tatsache, daß die Abspaltungsreaktionen stark exotherm verlaufen, dafür, daß der Übergangszustand näher beim tetraedrischen Ausgangszustand als beim koplanaren Endzustand liegt. Mit dieser Auffassung stehen ferner die Beobachtungen von Jones und Vernos ${ }^{11}$ im Einklang, wonach bei bimolekularen nukleophilen Substitutionen an Doppelbindungen die vorhandene cis- oder transKonfiguration des Ausgangsproduktes erhalten bleibt, was nur durch einen nahezu tetraedrischen Ubergangszustand erklärt werden kann.

\section{Berechnung des Isomerenverhältnisses von 2- und 1-Olefin im Reaktionsprodukt der bimolekularen Abspaltung}

Die vorliegenden Rechnungen stützen sich auf die experimentellen Ergebnisse von Hughes, Ingold und Mitarbb. ${ }^{2}$ sowie von Brown und Mitarbeitern ${ }^{5}$. Die von diesen Autoren gefundenen Isomerenverhältnisse im Reaktionsprodukt der bimolekularen Abspaltung geben das Verhältnis der Geschwindigkeitskonstanten der konkurrierenden Abspaltungsrichtungen wieder. Die Berechnung dieses Verhältnisses erfordert eine Kenntnis des Unterschiedes der Aktivierungsenergien; hierfür genügt es, die sterischen Verteilungsfunktionen der isomeren Übergangszustände zu ermitteln, da diese aus demselben Ausgangsprodukt hervorgehen. An Stelle von Gl. (7) der 1. Mitt. ${ }^{1}$ ist daher der einfachere Ausdruck

$$
\ln k_{\mathrm{b}} / k_{\mathrm{a}}=\Delta \ln Q^{\mathrm{st}^{\prime}}-\Delta E^{\mathrm{st}} / R T
$$

zu verwenden, wobei

und

$$
\Delta \ln Q^{s \mathrm{t}^{\prime}}=\ln Q_{\mathrm{b}^{\mathrm{st}}}-\ln Q_{\mathrm{a}}{ }^{\mathrm{st}}
$$

$$
\Delta E^{\mathrm{st}}=E_{\mathrm{b}}{ }^{\mathrm{st}}-E_{\mathrm{a}} \mathrm{st}^{\mathrm{st}}
$$

ist und die Indices b und a sich auf die Übergangszustände für die Bildung des 2-Olefins bzw. des 1-Olefins beziehen.

Gl. (1) bedarf jedoch noch einer Ergänzung, da die während des Abspaltungsprozesses entstehende Doppelbindung durch benachbarte Alkylgruppen .eine Stabilisierung erfährt. Die Zahl dieser Alkylgruppen ist bei den 2-Olefinen stets größer als bei den 1-Olefinen; der hierdurch bedingte Unterschied der Stabilisierungsenergien wird mit $\Delta E^{\mathrm{stab}}$ bezeichnet. Auf Grund der gemessenen Hydrierungswärmen ${ }^{12}$ hat dieser Stabilisierungseffekt für eine Methyl-, Äthyl-, Isopropyl- und t-Butylgruppe nahezu den gleichen Wert von rund 2,5 kcal/Mol. Man kann dies durch eine Überlagerung von Hyperkonjugations- und Induktionseffekt erklären, wobei ersterer in der genannten Reihenfolge linear abnimmt, während letzterer in der gleichen Reihenfolge linear ansteigt. Nach Dewar und Schmeising ${ }^{13}$ rührt jedoch die Stabilisierung von einer Erhöhung der Bindungsenergie der $\mathrm{C}-\mathrm{C}$-Bindung beim Utbergang

10 R. B. Turner, D. E. Nettleton u. M. Perelman, J. Amer. chem. Soc. 80, 1430 [1958].

11 E. Jones u. C. A. Vernon, Nature [London] 176, 791 [1955].

12 J. B. Conant u. G. B. Kistiakowsky, Chem. Reviews 20, 181 [1937].

13 M. J.S. Dewar u. H. N. Schmeising, Tetrahedron 5, 166 [1959]. 


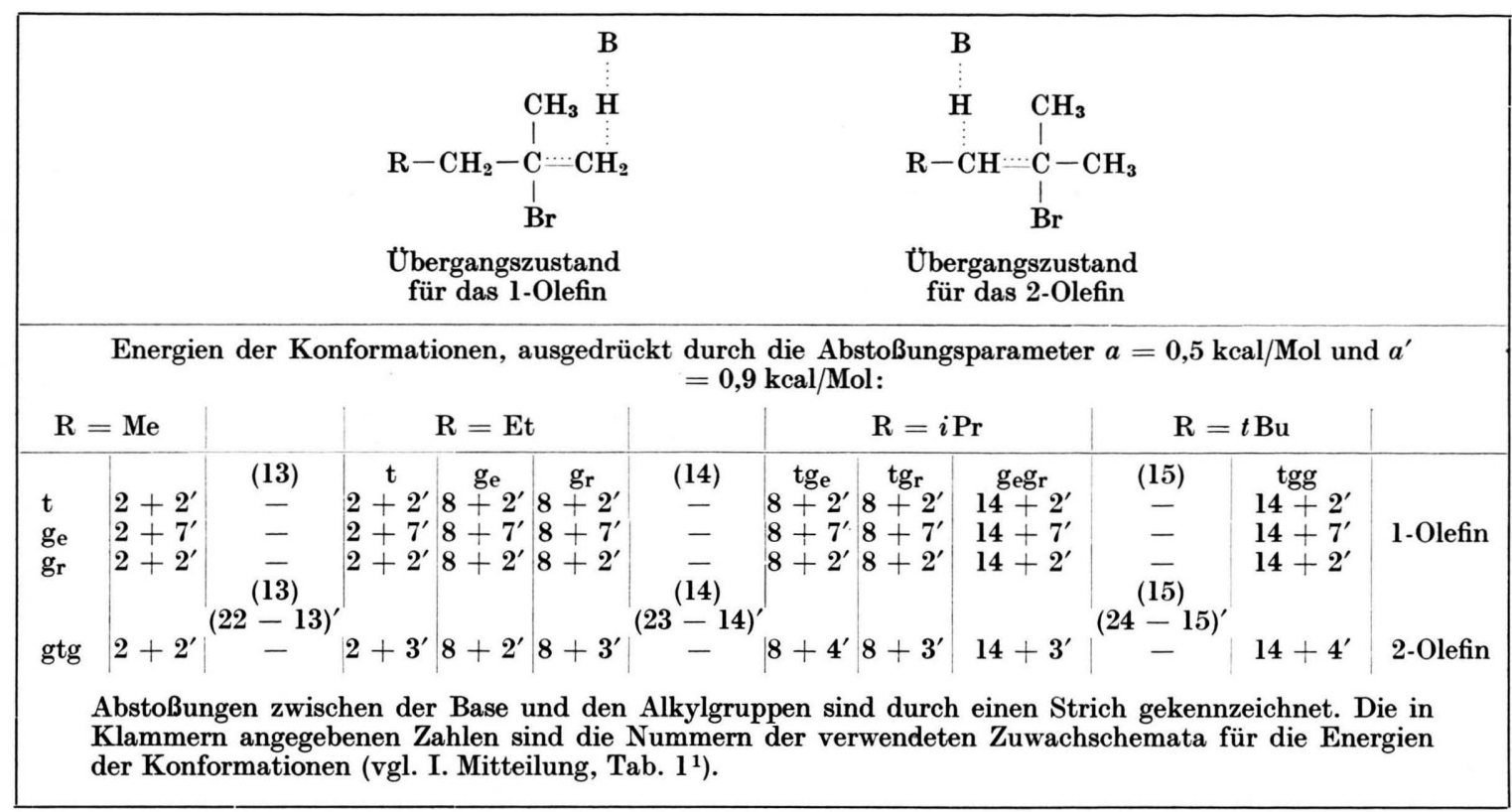

Tab. 1. Ermittlung der sterischen Energien der Konformationen der beiden isomeren Übergangszustände für die Bildung des 1- und 2-Olefins im Falle der bimolekularen $\mathrm{HBr}$-Abspaltung aus tertiären Alkylbromiden $\mathrm{R}-\mathrm{CH}_{2}-\mathrm{CBr}\left(\mathrm{CH}_{3}\right)_{2} ; \mathrm{Anwendung}$ des Aufbauverfahrens.

eines der C-Atome aus der $\mathbf{s p}^{\mathbf{3}}$-Hybridisierung in die $\mathrm{sp}^{2}$-Hybridisierung her, woraus sich die Unabhängigkeit von der Natur der Alkylgruppe ohne weiteres ergibt. Um bei den vorliegenden Rechnungen die sterischen Effekte klar hervortreten zu lassen, wurde in Anlehnung an Dewar und Schmeising ${ }^{13}$ stets für alle 4 genannten Alkylgruppen dieselbe Stabilisierungsenergie eingesetzt.

Wie ebenfalls aus den Hydrierungswärmen ${ }^{12}$ hervorgeht, nimmt der Stabilisierungseffekt nicht ganz linear mit der Zahl der Alkylgruppen an derselben Doppelbindung zu, selbst wenn man berücksichtigt, daß zwischen zwei $c i s$-ständigen Methylgruppen eine sterische Abstoßungsenergie von $1,0 \mathrm{kcal} / \mathrm{Mol}$ herrscht. So erhöht sich die Stabilisierungsenergie beim Übergang vom 1-Buten zum trans-2-Buten um $2,7 \mathrm{kcal} / \mathrm{Mol}$, beim Übergang vom Isobutylen zum Trimethyläthylen um $1,5+1,0=2,5 \mathrm{kcal} / \mathrm{Mol}$, beim Übergang vom Trimethyläthylen zum Tetramethyläthylen jedoch nur noch um $0,3+1,0=1,3 \mathrm{kcal} / \mathrm{Mol}$. Außerdem kann sich dieser Einfluß der Alkylgruppen auf die entstehende Doppelbindung im Übergangszustand noch nicht voll auswirken, so daß in die Rechnungen nur etwa $2 / 3$ der für die fertigen Olefine gültigen Beträge als Stabilisierungsenergien eingesetzt wurden, und zwar beträgt $\Delta E^{\text {stab }}$ für die sekundären Alkylderivate (Tab. 4 und 6) 2,0 kcal/
Mol (statt 2,7 kcal/Mol), für die tertiären Alkylderivate (Tab. 2 und 5) $1,8 \mathrm{kcal} / \mathrm{Mol}$ (statt $2,5 \mathrm{kcal} /$ Mol) und für die tertiären Alkylderivate mit einer $\beta$-ständigen Methylgruppe (Tab. 3) $2,4 \mathrm{kcal} / \mathrm{Mol}$ (statt $2,5+1,3=3,8 \mathrm{kcal} / \mathrm{Mol}$ ).

Weiterhin ist es notwendig, einen statistischen Faktor $P$ einzuführen, der die Zahl der für den Angriff der Base verfügbaren gleichwertigen H-Atome bei der Bildung des jeweiligen Übergangszustandes berücksichtigt. Für die Bildung des l-Olefins hat $\boldsymbol{P}_{\mathrm{a}}$ bei den tertiären Alkylderivaten (Tab. 2, 3 und 5) den Wert 6, bei den sekundären (Tab. 4 und 6) den Wert 3; für die Bildung des 2-Olefins hat $\boldsymbol{P}_{\mathrm{b}}$ bei den tertiären Alkylderivaten die Werte 2 (Tab. 2 und 5) bzw. 1 (Tab. 3), bei den sekundären (Tab. 4 und 6) für die trans- und die cis-Verbindung jeweils den Wert 1. An die Stelle von Gl. (1) tritt daher der vollständigere Ausdruck:

$$
\ln k_{\mathrm{b}} / k_{\mathrm{a}}=\Delta \ln Q^{\mathrm{st}}-\Delta E^{\mathrm{st}} / R T+\Delta E^{\mathrm{stab}} / R T-\Delta \ln P,
$$

wobei

$$
\Delta \ln P=\ln P_{\mathrm{a}}-\ln P_{\mathrm{b}}
$$

ist.

\section{Ermittlung der Energien der Konformationen der isomeren Übergangszustände}

Bei der Aufstellung der sterischen Verteilungsfunktionen der Übergangszustände läßt sich die 


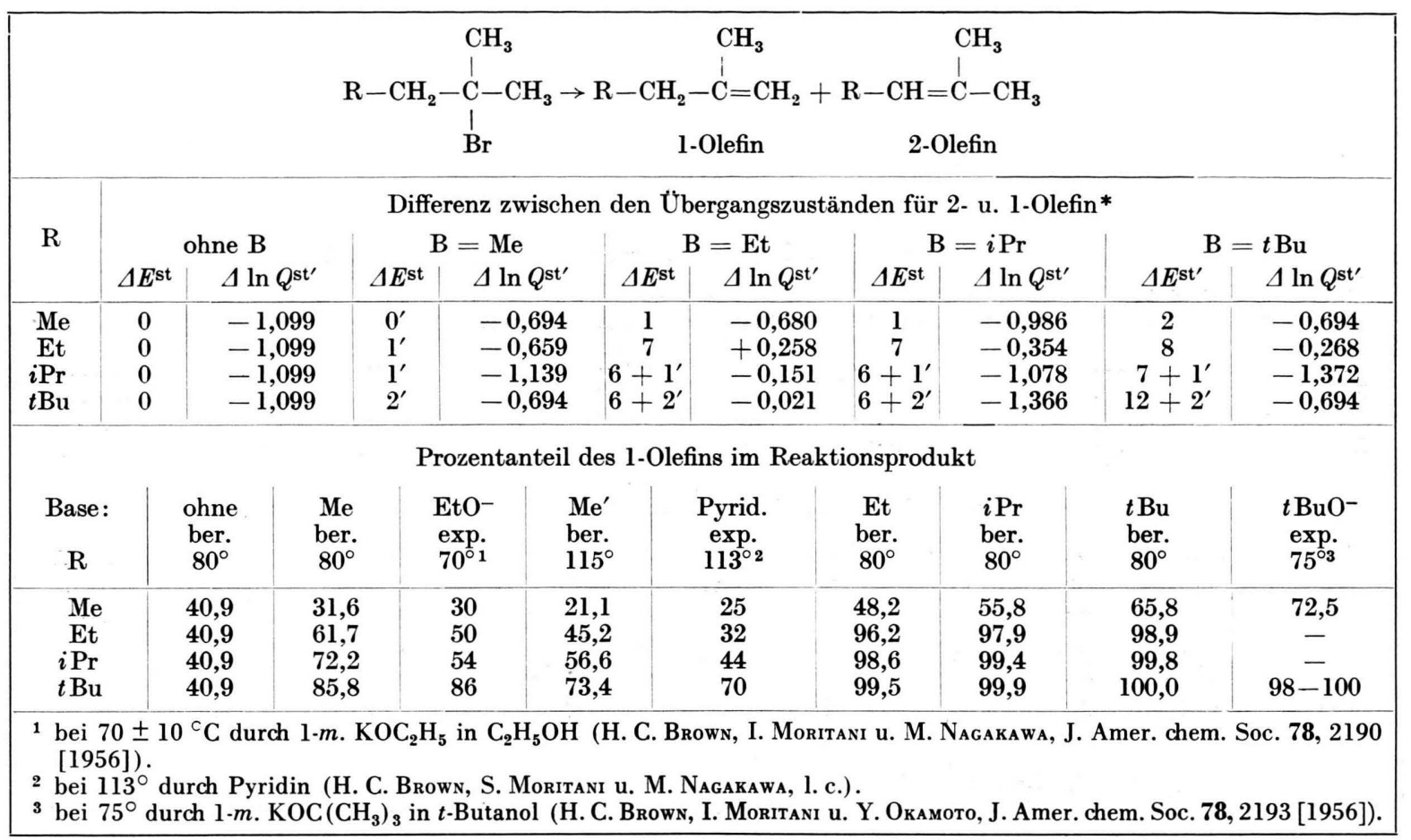

Tab. 2. Bimolekulare HBr-Abspaltung aus tertiären Alkylbromiden. Einfluß des Raumbedarfs des Alkylrestes R und der Base auf die Orientierung.

elektrophile Gruppe $\mathrm{X}$ in guter Näherung durch eine sterisch äquivalente Alkylgruppe ersetzen, nämlich das Br-Atom (Wirkungsradius 1,95 $\AA$ ) durch eine Methylgruppe (Wirkungsradius 2,0 ̊), die Dimethylsulfonium-Gruppe $-\mathrm{S}^{\oplus}\left(\mathrm{CH}_{3}\right)_{2}$ durch eine Isopropyl-Gruppe und die TrimethylammoniumGruppe $-\mathrm{N}^{\oplus}\left(\mathrm{CH}_{3}\right)_{3}$ durch eine $t$-Butylgruppe. Genaue Angaben über den Raumbedarf der Base in der Nähe des Reaktionszentrums im Übergangszustand können dagegen nicht gemacht werden. Im Experiment handelt es sich um das Äthylat- oder $t$-Butylat-Anion bzw. um Pyridin, die in trans-Stellung zur Gruppe $\mathrm{X}$ an das abzuspaltende H-Atom herantreten und damit im Übergangszustand eine ähnliche Rolle einnehmen wie ein neuer Substituent. Aus diesem Grund wird die Base wie ein Methylseitenzweig der Kette behandelt, jedoch unter Verwendung eines besonderen Abstoßungsparameters von $a^{\prime}=0,9 \mathrm{kcal} / \mathrm{Mol}$ zwischen dem Reaktionszentrum der Base und den übrigen Substituenten des Moleküls. Eine Untersuchung des Einflusses von Raumvergrößerungen der Base ist durch Anwendung des Kettenverlängerungs-Verfahrens möglich, was formal der Überführung einer Methylgruppe in eine Äthyl-, Isopropyl- oder $t$-Butylgruppe entspricht; hierbei wird wieder der normale AbstoBungsparameter von $a=0,5 \mathrm{kcal} / \mathrm{Mol}$ verwandt.

Tab. 1 gibt ein Beispiel für die Anwendung des in der 1 . Mitt. ${ }^{1}$ beschriebenen KettenverlängerungsVerfahrens zur Ermittlung der sterischen Energien der Konformationen der Übergangszustände. Wichtig ist dabei vor allem, daß mit dem Angriff der Base an einem der H-Atome von den 3 Konformationen um die entstehende Doppelbindung nur eine übrig bleibt, bei der sich Base und Gruppe $\mathrm{X}$ in transStellung befinden.

\section{Diskussion der Ergebnisse}

a) Einfluß des Raumbedarfs des Alkylrestes R. Wie aus den Spalten $B=M e$ der Tab. 2, 3 und 4 hervorgeht, hat die Vergrößerung des Raumbedarfs des Alkylrestes R, d. h. die Einführung von Substituenten am $\gamma$-C-Atom zum Halogen, eine systematische Verschiebung der Isomerenverhältnisse zugunsten des 1-Olefins zur Folge. Die Rechnungen lassen erkennen, daß hierfür in erster Linie die Zunahme der Differenzen $\Delta E^{\text {st }}$ verantwortlich ist, die von $0 \mathrm{a}(\mathrm{R}=\mathrm{Me})$ auf $2 \mathrm{a}^{\prime}$

* Die in den Tab. 2,3 u. 4 angegebenen Werte für $\Delta \ln Q^{\text {st' }}$ beziehen sich sämtlich auf $80^{\circ} \mathrm{C}$. 


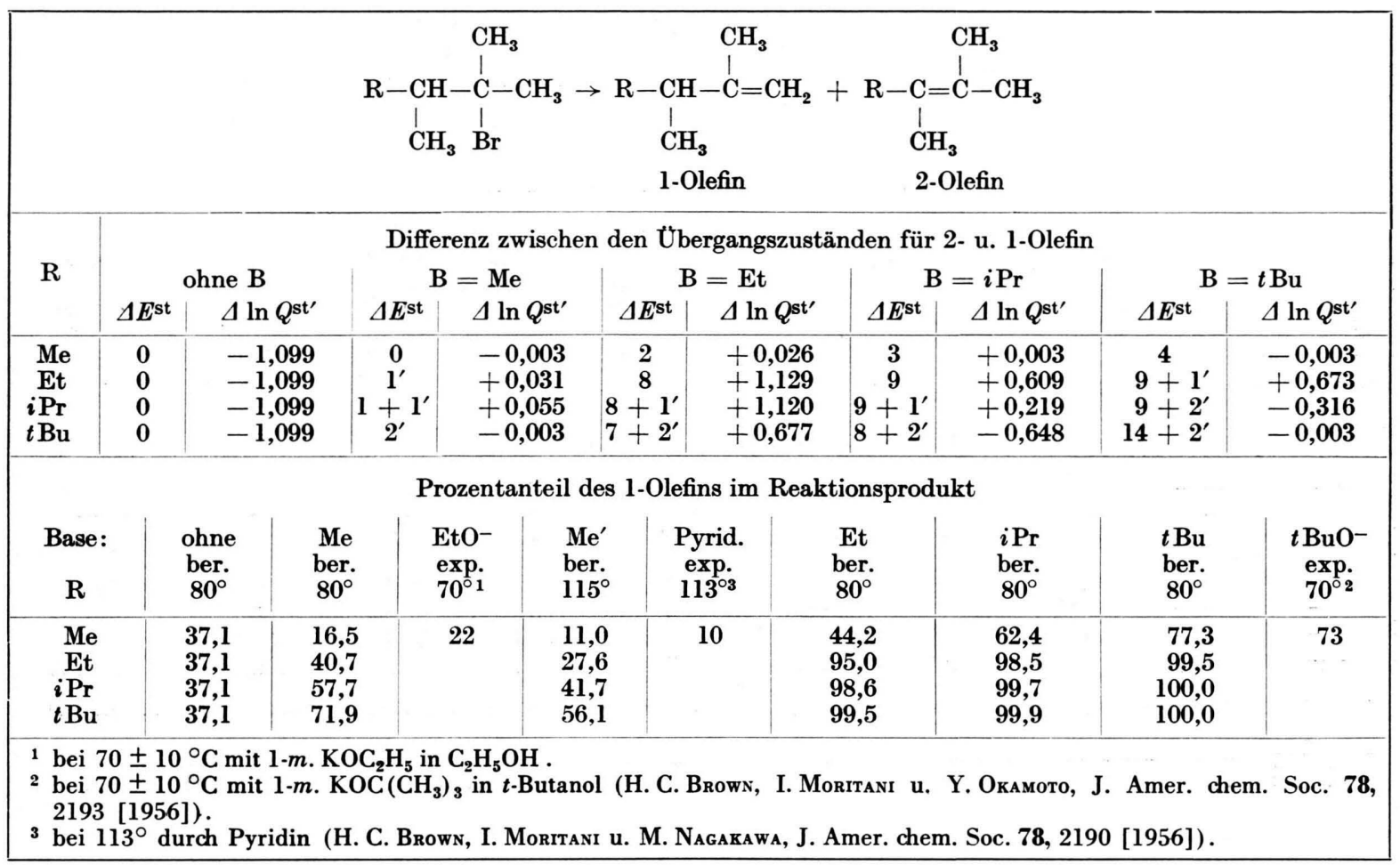

Tab. 3. Bimolekulare HBr-Abspaltung aus tertiären Alkylbromiden. Einfluß des Raumbedarfs des Alkylrestes R, einer $\beta$-ständigen Methylgruppe und der Base auf die Orientierung.

$(\mathrm{R}=t \mathrm{Bu})$ ansteigen. Während die Abstoßungen zwischen $R$ und der Base in der Reihenfolge $R=M e$, Et, $i \operatorname{Pr}, t \mathrm{Bu}$ im Übergangszustand für das 1-Olefin (vgl. Tab. 1) konstant bleiben, nehmen sie im Übergangszustand für das 2-Olefin zu. Qualitativ ist diese Tatsache verständlich, da im ersteren Fall die Base das 6. Atom der Kette bezüglich der Seitenzweige des Restes $R$ ist, im letzteren Fall jedoch das 4. Atom. Die sterischen Einflüsse von Verzweigungen reichen aber über das jeweilige 5 . Atom der Kette nicht hinaus (vgl. die 1. Mitt. ${ }^{1}$ ). Daneben spielen auch sterische Entropieeffekte eine gewisse Rolle; die Verschiebung des Isomerenverhältnisses beim Übergang vom 2-Methyl-2-brompentan (Tab. 2, $\mathrm{B}=\mathrm{Me}, \quad \mathrm{R}=\mathrm{Et}$ ) zum 2.4-Dimethyl-2-brompentan (Tab. 2, $\mathrm{R}=i \operatorname{Pr}$ ) z. B. ist lediglich auf eine Veränderung von $\Delta \ln Q^{\text {st }}$ zurückzuführen.

Wichtig ist auch die Feststellung, daß die Differenz $\Delta E^{\text {st }}$ bereits beim Übergang von $\mathrm{R}=\mathrm{Me} \mathrm{zu}$ $\mathrm{R}=\mathrm{Et}$, d. h. bei einer Kettenverlängerung am $\gamma$-CAtom zum Halogen, um eine Einheit wächst. Die hierbei beobachtete Verschiebung des Isomerenverhältnisses muß also in erster Linie auf einen sterischen Effekt zurückgeführt werden und nicht etwa auf einen verringerten Stabilisierungseffekt der Äthylgruppe an der Doppelbindung im Vergleich zur Methylgruppe.

Ein Vergleich der Spalten B= Me in den Tab. 2 und 3 zeigt, daß die Einführung einer Methylgruppe in $\beta$-Stellung zum Halogen praktisch keinen Einfluß auf die $\Delta E^{\text {st }}$-Werte ausübt. Die Summen $\Delta \ln Q^{\text {st' }}$ $-\Delta E^{\text {st }} / R T-\Delta \ln P$ sind in beiden Fällen sogar fast identisch, mit Ausnahme von $\mathrm{R}=i \mathrm{Pr}$, jedoch beträgt auch hier der Unterschied nur rund 0,2. Offenbar übt eine $\beta$-ständige Methylgruppe keinen nennenswerten sterischen Effekt auf das Isomerenverhältnis aus; lediglich der Stabilisierungseffekt beim 2-Olefin wird hierdurch vergrößert.

Angesichts der Feststellung, daß nur Substituenten am $\gamma$-C-Atom eine Erhöhung der $\Delta E^{\text {st }}$-Werte bewirken, wird auch verständlich, warum Hughes, Ingold und Mitarbb. ${ }^{2}$ die sterischen Effekte bei Abspaltungsreaktionen als vernachlässigbar ansahen; ihre Untersuchungen beschränkten sich nämlich auf $\alpha$ - und $\beta$-substituierte Alkylderivate.

Die HBr-Abspaltung aus sekundären Alkylbromiden (Tab. 4, Spalte $\mathrm{B}=\mathrm{Me}$ ) liefert ein Gemisch von 1-Olefin und trans- und cis-2-Olefin. Hier beeinflus- 


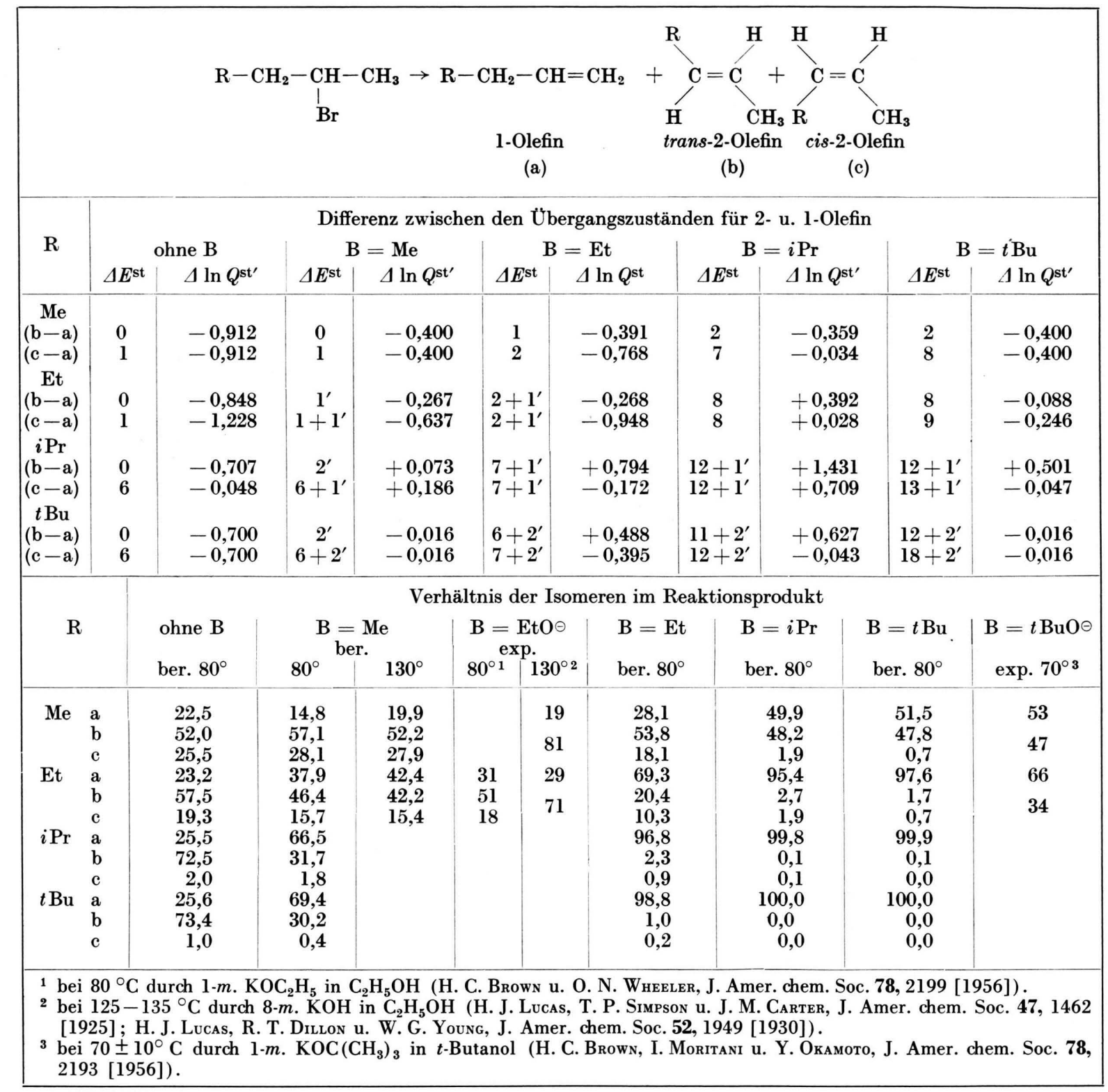

Tab. 4. Bimolekulare HBr-Abspaltung aus sekundären Alkylbromiden. Einfluß des Raumbedarfs des Alkylrestes R und der Base auf die Orientierung.

sen die sterischen Effekte sowohl das Verhältnis von 1-Olefin zu 2-Olefin wie auch das Verhältnis von trans- zu cis-2-Olefin. Die $\Delta E^{\text {st }}$.Werte zwischen 1-Olefin und trans-2-Olefin sind die gleichen wie bei den tertiären Alkylbromiden und rühren von Abstoßungen zwischen $\mathbf{R}$ und der Base $B$ her. Die erhöhten $\Delta E^{\text {st }}$-Werte zwischen 1-Olefin und cis-2-Olefin in den Fällen $\mathrm{R}=i \operatorname{Pr}$ und $\mathrm{R}=t \mathrm{Bu}$ sind durch eine zusätzliche sterische Spannung zwischen den Alkylgruppen im Übergangszustand für das 2-Olefin bedingt. b) Einfluß des Raumbedarfs der B a s e. Die Tab. 2, 3 und 4 lassen erkennen, daß der Raumbedarf der Base als Reaktionspartner von entscheidender Bedeutung für das Zustandekommen der sterischen Einflüsse auf die Orientierung ist. Vernachlässigt man nämlich den Raumbedarf der Base (Spalten „ohne B“), so bleibt auch die Vergrößerung des Alkylrestes ohne jeden Effekt, ganz im Gegensatz zu den experimentellen Befunden. Die als Voraussetzung für die ebene trans-Elimination 


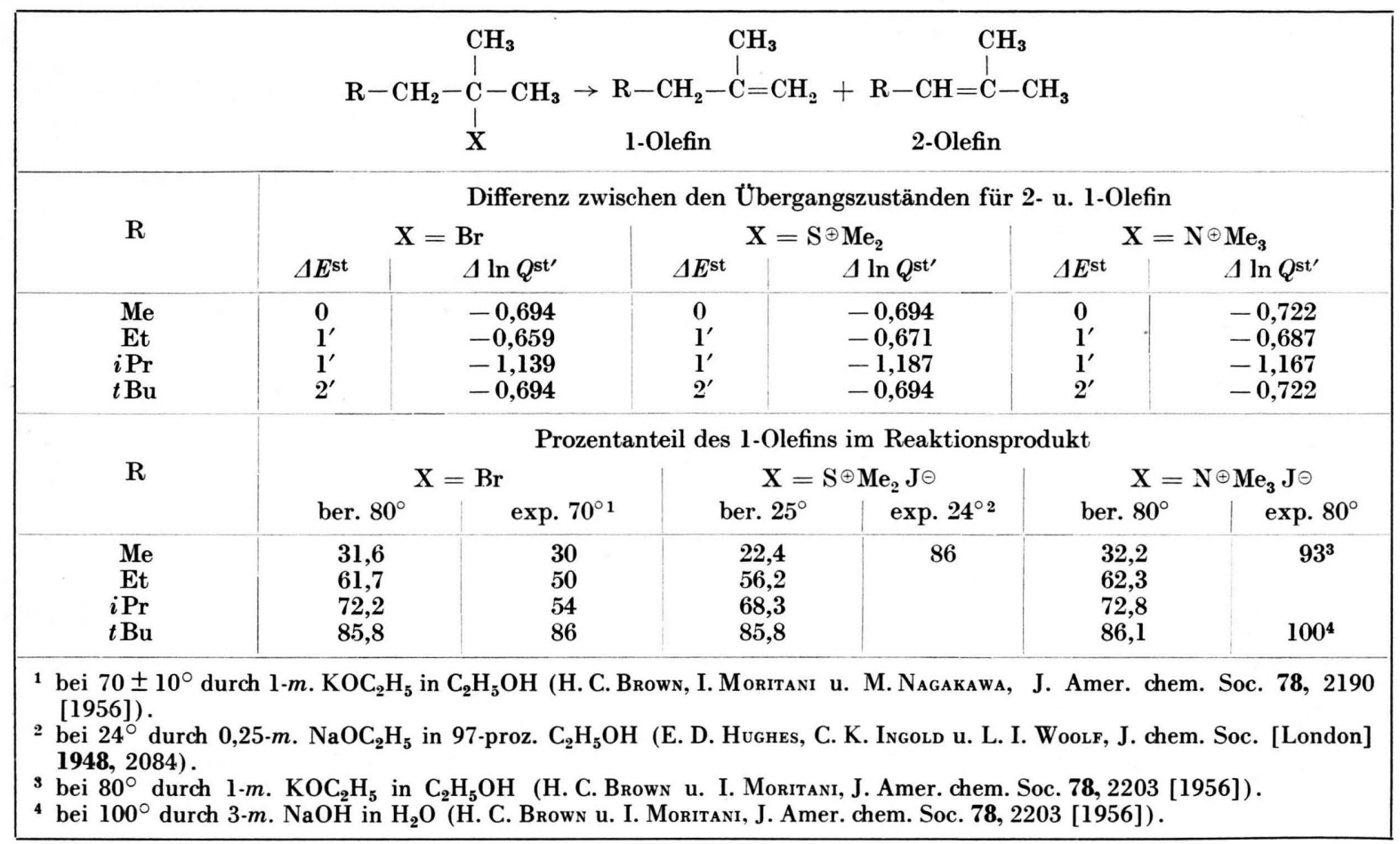

Tab. 5. Bimolekulare Abspaltungsreaktion aus tertiären Alkylderivaten. Einfluß des Raumbedarfs der elektrophilen Gruppe X auf die Orientierung. (Base $\mathrm{B}=\mathrm{Me}$ ).

erforderliche Rotationseinschränkung an der entstehenden Doppelbindung genügt offenbar alleine nicht, um die beobachteten Verschiebungen der Isomerenverhältnisse zu erklären. Beim Vergleich mit dem Experiment entspricht das Modell : Base $=$ Methylgruppe mit erhöhtem Abstoßungsparameter $a^{\prime}=0,9 \mathrm{kcal} / \mathrm{Mol}$ etwa den mit Äthylat oder Pyridin erhaltenen Ergebnissen, während das Modell Base $=t$-Butylgruppe ungefähr die beobachteten Resultate mit $t$-Butylat wiedergibt.

Sicherlich spielt bei den experimentellen Ergebnissen auch der Einfluß des Lösungsmittels eine gewisse Rolle, wie aus einem Vergleich der mit Kaliumäthylat in Äthanol und der mit Pyridin erhaltenen Isomerenverhältnisse hervorgeht (Tab. 2 und 3, untere Hälfte). Trotz der höheren Versuchstemperatur ist in Pyridin das 2-Olefin stärker begünstigt. Hierfür kann man eine sterische Hinderung der Solvatation des Übergangszustandes für das 2-Olefin verantwortlich machen, die sich in Alkohol stärker auswirkt als in Pyridin. Um diesem Effekt Rechnung zu tragen, wurden die Differenzen $\Delta E^{\text {stab }}$ in den Spalten $\mathrm{B}=\mathrm{Me}^{\prime}$ (ber. $115^{\circ}$ ) um $0,6 \mathrm{kcal} / \mathrm{Mol}$ erhöht, d. h. auf 2,4 kcal/Mol in Tab. 2 und auf $3,0 \mathrm{kcal} / \mathrm{Mol}$ in Tab. 3; die Abstoßungsparameter wurden hingegen unverändert beibehalten. Teilweise wird der Einfluß einer sterischen Hinderung der Solvatation schon bei der Aufstellung der sterischen Verteilungsfunktion erfaßt, da Konformationen, in denen die Annäherung des
Lösungsmittels an das Reaktionszentrum behindert ist, sich gleichzeitig durch eine erhöhte sterische Energie auszeichnen.

c) EinfluB des Raumbedarfs der elektrophilen Gruppe X. Die Ergebnisse für die bimolekulare Abspaltungsreaktion aus tertiären und sekundären Alkylbromiden, Alkyldimethylsulfonium- und Alkyltrimethylammonium-Salzen sind in den Tab. 5 und 6 zusammengestellt. Hiernach sind die für die sterischen Effekte in erster Linie maßgeblichen Differenzen $\Delta E^{\text {st }}$ vom Raumbedarf der elektrophilen Gruppe $\mathrm{X}$ unabhängig, so daß sich kaum nennenswerte Veränderungen der Isomerenverhältnisse ergeben. Die qualitative Begründung für dieses Resultat besteht darin, daß die bei der Verlängerung der Gruppe $\mathrm{X}$ im Modell von $\mathrm{X}=\mathrm{Me}$ zu $\mathrm{X}=i \operatorname{Pr}$ und $\mathrm{X}=t \mathrm{Bu}$ hinzukommenden neuen Seitenzweige sowohl im Übergangszustand für das 1-Olefin wie auch im Übergangszustand für das 2-Olefin jeweils das 5-Atom der Kette bezüglich der Base bilden. Weil aber zwischen Base und Gruppe X eine gestreckte trans-Konfiguration vorausgesetzt wird, besteht in diesem Fall zwischen dem 1. und dem 5. Glied der Kette keine sterische Wechselwirkung mehr. 


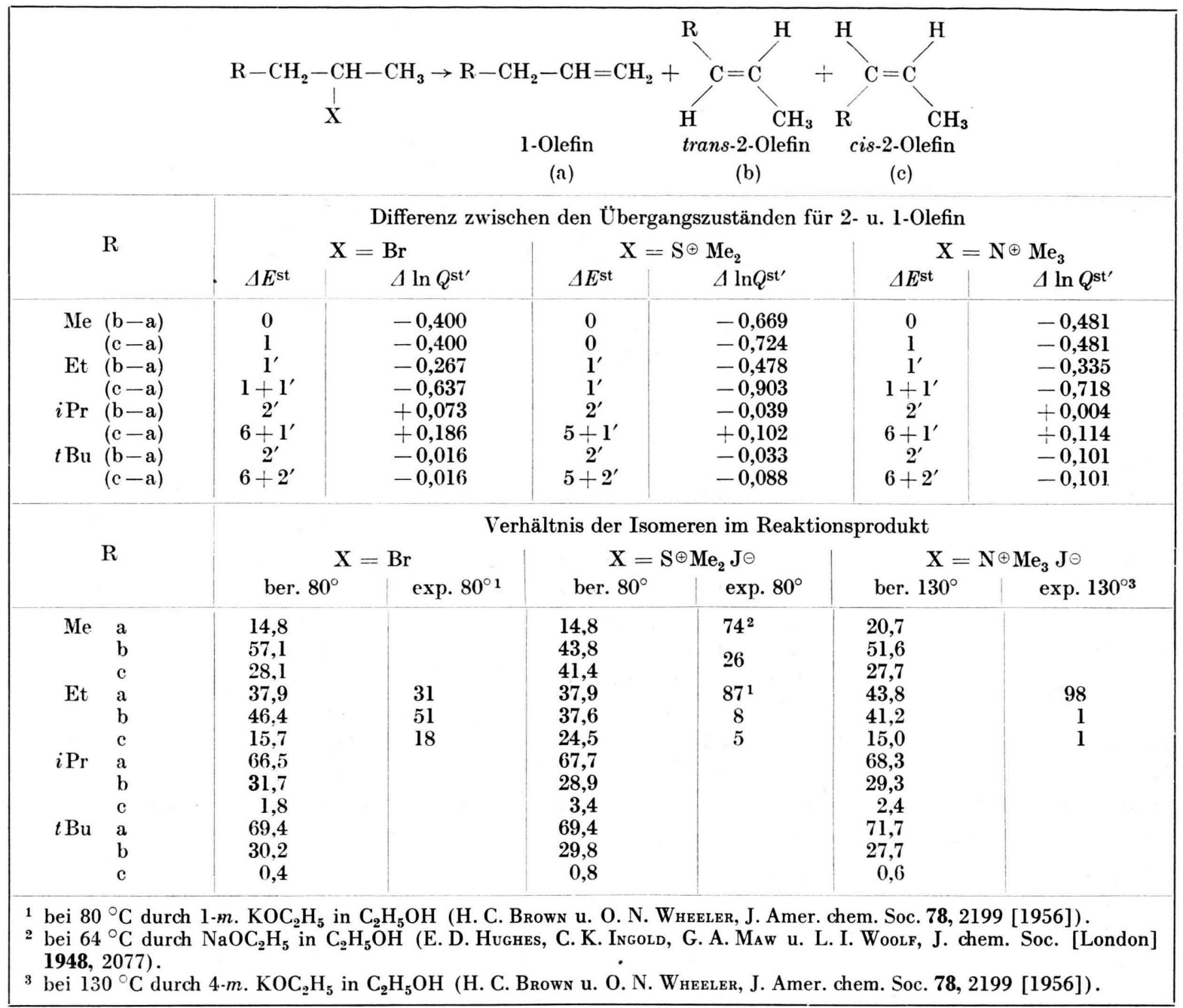

Tab. 6. Bimolekulare Abspaltungsreaktion aus sekundären Alkylderivaten. Einfluß des Raumbedarfs der elektrophilen Gruppe $\mathrm{X}$ auf die Orientierung. (Base $\mathrm{B}=\mathrm{Me}$ ).

Experimentell wird dagegen beim Übergang von den Bromiden zu den Dimethylsulfonium- und Trimethylammonium-Salzen eine starke Verschiebung des Isomerenverhältnisses zugunsten des 1-Olefins beobachtet. Die durchgeführten Rechnungen zeigen, daß im Rahmen des verwendeten Modells hierfür kein sterischer Effekt verantwortlich gemacht werden kann. Als Erklärung muß vielmehr - in Übereinstimmung mit der Ansicht von Hughes, Ingold und Mitarbb. ${ }^{2}$ - der von den geladenen Resten ausgehende starke Induktionseffekt herangezogen werden, der in die Rechnungen nicht einbezogen wurde. BRown ${ }^{5}$ hat zwar gefunden, daß auch durch bestimmte ungeladene Reste $\mathrm{X}$ eine Umkehr der $\mathrm{Ab}$ spaltungsrichtung von der $\mathrm{S}$ a y $\mathrm{z}$ eff schen zur
$\mathrm{H}$ of $\mathrm{m}$ a $\mathrm{n} n$ schen Regel herbeigeführt werden kann, jedoch sind auch diese Gruppen wesentlich stärker induktiv wirksam als Halogenatome.

Angesicht des verwendeten einfachen Modells für die sterischen Effekte kann die erreichte Übereinstimmung der Rechnungen mit den experimentellen Befunden als durchaus befriedigend bezeichnet werden. Wichtiger sind jedoch noch die qualitativen Ergebnisse der Theorie, da sie verallgemeinerungsfähige Aussagen über die Bedingungen erlauben, unter denen mit sterischen Einflüssen auf die Orientierung der bimolekularen Abspaltungsreaktion zu rechnen ist.

Dem Direktor des Instituts, Herrn Professor Dr. N. G. Schmahl, danke ich für das der vorstehenden Arbeit entgegengebrachte fördernde Interesse. 